\title{
Identifying Test Items That Perform Differentially in Population Subgroups: A Partial Correlation Index
}

\author{
Lawrence d. Stricker \\ Educational Testing Service
}

\begin{abstract}
Verbal items on the GRE Aptitude Test were analyzed for race (white vs. black) and sex differences in their functioning, using a new procedure-item partial correlations with subgroup standing (race or sex), controlling for total score-as well as two standard methods-comparisons of subgroups' item characteristic curves and item difficulties. The partial correlation index agreed with the item characteristic curve index in the proportions of items identified as performing differentially for each race and sex. These two indexes also agreed in the particular items that they identified as functioning differentially for the sexes, but not in the items that they identified as performing differently for the races. The partial correlation index consistently disagreed with the item difficulty index in the proportions of items identified as functioning differentially and in the particular items involved. The items identified by the partial correlation index as performing differentially, like the items identified by the other indexes, generally did not differ in type or content from items not so identified, with one major exception: this index identified items with female content as functioning differently for the sexes.
\end{abstract}

Test bias, whether associated with race, sex, or other population subgroups, is a highly complex issue. Bias, in principle, can manifest itself in a variety of ways, and little consensus exists about what constitutes acceptable evidence of its exis-

APPLIED PSYCHOLOGICAL MEASUREMENT

Vol. 6, No. 3. Summer 1982, pp. 261-273

(C) Copyright 1982 Applied Psychological Measurement Inc. $0146-6216 / 82 / 030261-13 \$ 1.65$ tence or about what are the best means of dealing with it (Flaugher, 1978). The problem of minimizing test bias has been attacked at both the score and the item level. One promising approach is to identify, during the course of test construction, items that perform differently for the relevant subgroups, the presumption being that these items are biased. Such items can then be inspected and, if need be, excluded from the test.

\section{I}

A variety of procedures has been proposed for this purpose (see the reviews by Jensen, 1980; Merz, 1978; Petersen, 1980; Rudner, Getson, \& Knight, 1980; Shepard, 1981). All these methods have two major features in common: (1) they assume that the test is unidimensional and that the total score (or the typical item) is relatively free of differential functioning, and (2) they rely on internal analyses of the test to identify deviant items.

Item difficulty. The best known and most widely used procedure involves plots of the corresponding item difficulty values for subgroups, outlying items being considered as performing differently (e.g., Angoff \& Ford, 1973). This method controls for overall differences in the ability of the subgroups by comparing the items with each other; items are identified that diverge 
in their difficulty for the subgroups and, hence, favor one of them.

The drawback to this approach is that items are not uniform in every respect; they differ in characteristics that, in and of themselves, produce divergencies in difficulty. Items with greater discriminating power will invariably diverge more; and those with greater difficulty will diverge more whenever the subgroups differ in their tendency to guess.

This index is computed, as outlined by Angoff and Ford (1973), by converting item difficulties for each subgroup to normal deviates and, in turn, linearly transforming them to "deltas." The pair of delta values for each item are plotted on a graph and a line of best fit, corresponding to the main axis, is calculated. The perpendicular distance of the location of each item from this line is then determined. This distance, which represents the item's divergence, is conventionally considered to be significant if it exceeds a fixed number of standard deviations of the distance values for the set of items. These distances will tend to have (1) a mean of zero, because they are deviations from a line of best fit; and (2) a normal distribution, providing that the distributions of deltas for the two subgroups are bivariate normal.

Item characteristic curve. Perhaps the soundest approach, in principle, compares the subgroups' item characteristic curves (ICCs) for each item, the items with divergent curves being seen as functioning differentially (e.g., Lord, 1977a). This method controls for subgroup differences in overall ability by comparing the difficulty of an item for examinees from the different subgroups who are at the same ability level; items are identified that consistently favor a subgroup at various levels or that favor one subgroup at a particular level of ability and favor the other subgroup at a different level.

One problem with this method is that the extreme regions of the curves are unstable when the score distributions for the subgroups are considerably different. Unreliability in the total scores also affects this procedure. A practical limitation is that each subgroup must be very large (perhaps 1,000 or more).

As described by Lord (1980), this index is obtained by computing the ICC for each item with the LOGIST program (Wood, Wingersky, \& Lord, 1976) for a three-parameter model. Each item's guessing (c) parameter is determined from the pooled data for the two or more samples being compared, its discrimination (a) and difficulty $(b)$ parameters are estimated separately for each sample, and all of the $b$ parameters are put on the same scale. The significance of the difference between the curves for a pair of samples is then assessed by an approximate $\chi^{2}$ test that simultaneously evaluates discrepancies in both $a$ and $b$ parameters.

Partial correlation. Another index, though promising, has not been employed previously in this connection. This index, suggested by Lord $(\mathbb{1 9 7 7 b})$, is analogous to the partial correlation proposed by Darlington (1971) as one definition of test bias at the score level. Darlington argued that a test can be considered as biased if it has a significant partial correlation with subgroup standing (e.g., white vs. black) when the criterion is held constant. The present index is the item's partial correlation with subgroup standing, total score being held constant. Items with significant correlations are interpreted as performing differentially. This procedure, like the ICC index, controls for subgroup differences in overall ability. However, unlike the ICC index, this index compares the general difficulty of the item for the subgroups, essentially averaging differences between them at the various levels of ability; items are identified that consistently favor one subgroup.

This index is free of the major problems associated with other methods, though it shares the assumptions about the unidimensionality of the test and the lack of differential functioning in the total score. It also has several practical advantages: the index is simple to compute, applies to comparatively small samples (perhaps as few as 400 if sampling fluctuations in this index are to be reduced to a minimum), is flexible (any 
number of different kinds of subgroups can be accommodated simultaneously; and the subgroups can be represented by dummy-coded categories, e.g., race, or quantitative variables, e.g., socioeconomic status), provides for a significance test, and permits a straightforward evaluation of the size of effect. The main drawback of this procedure is that, in contrast to the ICC index, it cannot identify items which vary in their functioning in the subgroups at different levels of ability, favoring a subgroup at one point and penalizing it at another.

Operationally, the partial correlation index is computed by the following formula:

$$
r_{i S \cdot I_{\infty}^{\infty}}=\frac{r_{i S}-r_{i T \infty \infty} r_{T \infty S}}{\sqrt{1-r_{i T \infty}^{2}} \frac{\sqrt{1-r_{T \infty S}^{2}}}{\sqrt{1-}}},
$$

where

$r_{i s}$ is the correlation between the item responses (incorrect or omit $=0$, correct $=1$ ) and subgroup standing (e.g., for race, white $=0$, black $=1$; for sex, male $=0$, female $=1$ );

$\gamma_{i T \infty}$ is the correlation between the item response and the total score, adjusted for item overlap (the item is removed from the score in calculating the correlation) and corrected for attenuation in the score; and

$r_{r o s}$ is the correlation between the total score and subgroup standing, corrected for attenuation in the former.

All these correlations are product-moment coefficients. In the common case where subgroup standing is a dichotomous variable, and hence the correlation between the item and subgroup standing is a phi coefficient, the significance of this partial correlation can be determined with the special test of significance for a phi coefficient, $\chi^{2}=N \phi^{2}$, evaluated with one degree of freedom (Guilford, 1965). This test is an approximation, because variables are not only par- tialed out of this phi coefficient but also corrected for attenuation. In other situations, the significance of this partial correlation can be approximated with the standard test of significance for a partial correlation coefficient.

Two points should be noted about this index. First, the indexes for a set of items will tend to be normally distributed around a mean of zero, providing that the total score represents the only variance common to the items and subgroup standing, for this partial correlation is akin to the residual value in a factor matrix of the items and the subgroup variable after extraction of a factor representing the total score (Harman, 1976). Second, the magnitude of the indexes for different items cannot be precisely compared, because this measure is based on correlations for a dichotomous item, and these, in turn, are limited by the proportions passing and failing it (Lord \& Novick, 1968; Magnusson, 1967). This problem does not affect the significance of the index, and hence items can be directly compared with regard to their significance (see Appendix).

\section{Stadies of Aroility Iterms}

A number of studies have examined race and sex differences in the performance of ability test items, using many kinds of statistical indexes (see the review by Jensen, 1980). Although these investigations are necessarily limited by the problems in the methods that they employed, consistent findings - by virtue of the differences in item pools, populations, and specific details of the analytic procedures involved-can be interpreted, at least tentatively, as substantive results not attributable to statistical artifacts.

Much of the research has concerned the Preliminary Scholastic Aptitude Test (PSAT; Donlon \& Angoff, 1971), Scholastic Aptitude Test (SAT; Donlon \& Angoff, 1971), and Graduate Record Examinations (GRE) Aptitude Test (Conrad, Trismen, \& Miller, 1977), which are similar in the types of items used and their content. Studies of the PSAT are few in number and limited in scope. Negligible evidence of differ- 
ential functioning for whites and blacks was observed in overall analyses of this test (Angoff \& Ford, 1973; Cleary \& Hilton, 1968). Individual items were not examined, and differential performance for males and females was not investigated.

The differential functioning of items for whites and blacks as well as for males and females has been extensively studied on the SAT. Most studies identified few items that performed differently (e.g., Coffman, 1961). However, a noteworthy investigation by Marco and Lord (Lord, 1977a), the only one using the ICC index, identified a large number of verbal items that functioned differently for whites and blacks. The findings about the items uncovered in these studies are sparse. In general, items identified as performing differentially for whites and blacks or for males and females did not favor one race or sex. A consistent exception is that discrete verbal items (e.g., antonyms, analogies) with practical content favored males and those with human relationships content favored females (e.g., Coffman, 1961).

Race and sex differences in item functioning have also been investigated on the GRE Aptitude Test, but only to a limited extent. As in the case of the SAT research, these investigations identified few items and uncovered very little about their characteristics (e.g., Donlon, Hicks, \& Wallmark, 1980). The major study (Donlon et al., 1980) found that discrete verbal items with human relationships content and reading comprehension items with argumentative content favored females and that reading comprehension items with science content and data interpretation items favored males.

\section{Pripropese}

The aim of this study was to explore the usefulness of the partial correlation index in identifying race (white vs. black) and sex differences in the performance of verbal items on the GREAptitude Test. Specifically, the goals were (1) to determine whether differentially functioning items can be identified by this procedure; (2) to assess whether they are the same as those identified by the item difficulty and ICC indexes; and (3) to attempt to ascertain characteristics that distinguish the items identified by the various methods.

\section{INethod}

\section{Test Forreri and 面ems}

The 80 operational items in the Verbal section of the GRE Aptitude Test (Form ZGR2) were analyzed. This form was chosen because it contains reading passages with black as well as female content and because data are available for a large number of examinees, the form having been used in several administrations, beginning in October 1977.

\section{Samples}

Samples of whites and blacks (excluding Chicanos, Puerto Ricans, and other Hispamics) of both sexes were drawn from the 57,458 examinees who took this form of the test in the October 1977 and January 1978 administrations. In order to minimize extraneous influences of educational background and test-taking experience, the samples were limited to examinees with the characteristies listed below.

1. Never took the GRE Aptitude Test previously.

2. Had no test irregularity (e.g, examinee did not request cancellation of scores).

3. Undergraduate major in social sciences. ${ }^{1}$

4. Never attended graduate school full time.

\footnotetext{
'The BIQ classification of social science majors was used: American Studies, Anthropology, Business and Commerce, Communications, Economics, Education (including M.A. in Teaching), Educational Administration, Educational Psychology, Geography, Government, Guidance and Counseling, History, Industrial Relations and Personnel, International Relations, Journalism, Law, Library Science, Physical Education, Political Science, Psychology, Public Adminis* tration, Slavic Studies, Social Psychology, Social Work, Sociology, Urban Development (Regional Planning), and Other Social Sciences.
} 
5. Currently a senior or an unenrolled college graduate.

6. Native of United States (i.e., U.S. citizen, communicates best in English, and has domestic address).

This information was obtained from the Biographical Information Questions (BIQ) and registration form, completed when the examinees applied to take the test at these administrations, and from other records about these administrations and earlier ones.

Four basic samples were obtained for use in the main analyses of race and sex differences in item performance: white males $(N=1,122)$, white females $(N=1,471)$, black males $(N=284)$, and black females $(N=626)$. The two white samples comprised a randomly selected one-fourth of the qualified white examinees, and the black samples contained all the qualified black examinees.

Two pseudosamples, each the same size as an actual sample but composed of a different race or sex, were also obtained for supplementary analyses to verify the approximate significance tests for the partial correlation and ICC indexes. These nonoverlapping samples were randomly drawn from qualified white males not in the basic sample: (1) a pseudosample of black males $(N=284)$, of the same size as the basic sample of black males, for comparison with the basic sample of white males in the analyses of race differences in performance (i.e., the analogue of the analysis for white and black males, using two groups of white males); and (2) a pseudosample of black females $(N=626)$, of the same size as the basic sample of black females, for comparison with the pseudosample of black males in the analyses of sex differences in performance (i.e., the analogue of the analysis for black males and females, using two groups of white males). These two pseudosamples were selected because they corresponded to the smallest basic sample in each analysis and because this basic sample, when combined with the other sample in the analysis, had the smallest number of examinees. Verification of the significance tests is most critical for these relatively small samples.

\section{Item Indexes}

The partial correlation, item difficulty, and ICC indexes were computed as described previously. In calculating the partial correlation index, cases with a "not reached" response on an item were excluded in computing the three components of the index for that item. In calculating the ICC index, the data were pooled for the four basic samples in determining the item's $c$ parameter, and all the $b$ parameters were put on the scale for white males. The .05 level was used for the significance tests of the partial correlation and ICC indexes. For the item difficulty index, a distance was considered to be significant if it exceeded 1.96 standard deviations of the distances for the set of items in the same analysis. Because this criterion represents the 95 limits of a normal distribution, .05 of the distances in an analysis will be significant, providing only that the distances are normally distributed.

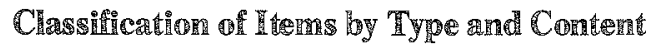

The items' type and content were categorized, using adaptations of the classification schemes employed in test construction. The items were classified into four types (Antonyms, Analogies, Sentence Completions, and Reading Comprehension) and six content areas (Aesthetic-Philosophical, World of Practical Affairs, Science, Human Relationships, Black, and Female).

\section{Statistical Analysis}

Parallel analyses of differential functioning were carried out for race and sex. Race differences in performance were analyzed separately for males and females; sex differences were analyzed separately for whites and blacks. The partial correlation, item difficulty, and ICC indexes were calculated in these analyses for all the items.

The correspondence between the items identified by the three indexes was assessed by unweighted Kappa coefficients (Cohen, 1960; Fleiss, Cohen, \& Everitt, 1969). For this pur- 
pose, items identified by the partial correlation and item difficulty indexes were classified as significant and positive (i.e., favoring blacks or females), significant and negative (i.e., favoring whites or males), or nonsignificant; and items identified by the $\mathbb{I C C}$ index were classified as significant or nonsignificant. However, in comparisons of the partial correlation or item difficulty indexes with the ICC index, the items identified by the first two indexes, like those identified by the last, were simply classified as significant or nonsignificant.

The association between the items identified by the indexes and the items' type or content was assessed by $\chi^{2}$ tests based on the classification of the items by type or content and by their significance, using the previously described categories (significant and positive, significant and negative, and nonsignificant for the partial correlation and item difficulty indexes; significant and nonsignificant for the ICC index).

\section{Results and Discussion}

\section{Main Analyses}

The number of items identified as significant or nonsignificant by the three indexes in the main analyses of differential functioning appear in Table 1 . The frequency distributions for the partial correlation index are given in Table 2.

In the analyses of race differences in performance, the partial correlation index identified as significant, for males as well as females, almost one-half of the items. Most of the indexes were less than .10 in absolute size, and only one exceeded .20. The indexes were about evenly distributed in sign, roughly one-half reflecting functioning that favors whites and the other representing functioning that favors blacks. The ICC index identified as significant about one-fourth of the items for each sex, a substantial but smaller proportion than that identified by the partial correlation index. The item difficulty index, in contrast to the others, identified as significant less than one-tenth of the items for males as well as females. About an equal num- ber of distances were positive and negative, onehalf representing performance favoring whites and the remainder reflecting performance favoring blacks.

In the analyses of sex differences in functioning, the partial correlation index identified as significant more items for whites than blacks. For whites, almost one-half the items were identified; for blacks, in comparison, only about one-quarter were identified. Most of the indexes were less than .10 in absolute size; only two were above .20 . The indexes were about evenly distributed in sign, one-half reflecting functioning that favors males and one-half representing functioning that favors females. The ICC index also identified as significant substantially more items for whites than for blacks-over one-third for the former and about one-fifth for the latter. The item difficulty index identified as significant fewer than one-tenth of the items for whites and blacks. The distances were about equally divided in sign, one-half representing performance favoring males and the other reflecting functioning that favors females.

One striking finding in both the race and sex analyses of differential functioning was the overall consistency with which substantial proportions of items were identified as significant across samples and across the partial correlation and ICC indexes. This agreement in the results for the two measures indicates that the unusually high proportions of items detected as performing differently by the indexes in this study - well above the level observed in all previous research, except Marco and Lord's (Lord, 1977a) investigation of race differences with the ICC index-cannot be attributed to the specific properties of the particular measure. Equally noteworthy, though, the results for the partial correlation index indicate that the effects involved were weak, judging from the magnitude of the indexes.

An important exception to this trend for sizable proportions of items to be identified as significant is the relatively small proportions identified in the analyses of sex differences for blacks. This divergence in the results for blacks 
Table 1

Identification of Items by Indexes in Main and Pseudo Analyses of Differential Functioning $(n=80)$

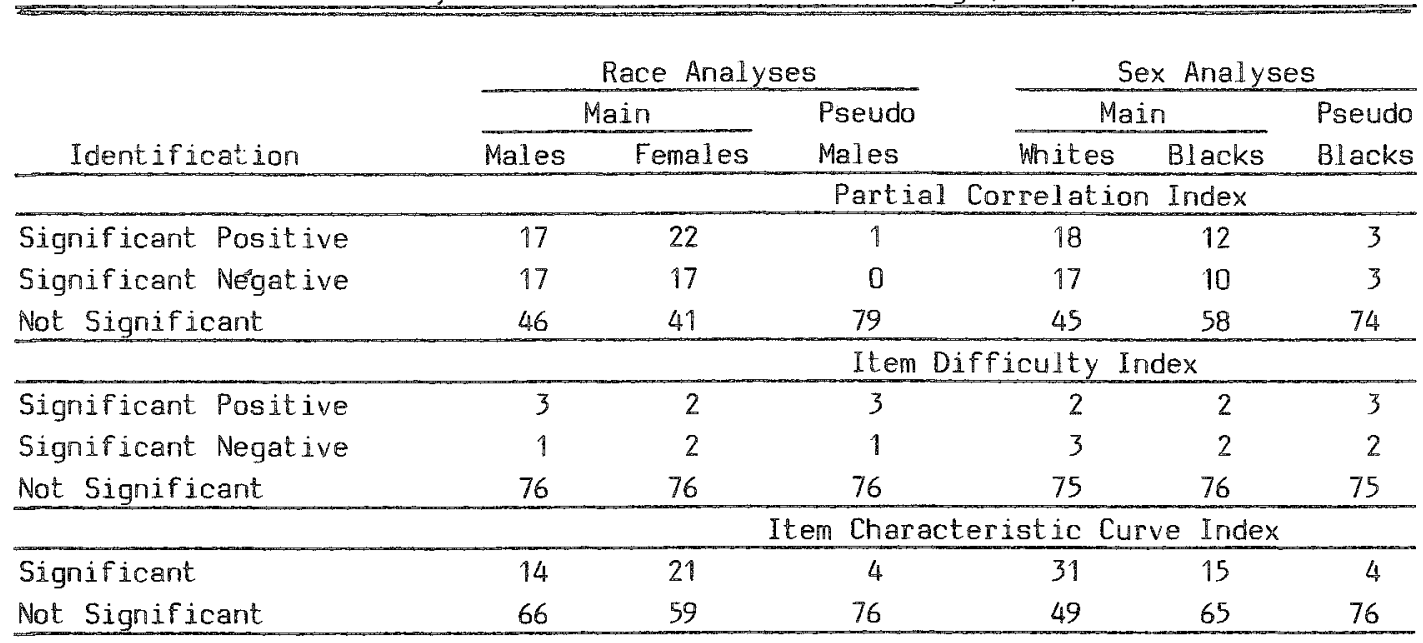

Note. In the race analyses, positive indexes signify items favoring blacks and negative indexes items favoring whites; in the sex analyses, positive indexes signify items favoring females and negative indexes items favoring males.

may be explainable, at least in part, by the smaller size of the black samples, which necessarily affects the magnitude of the partial correlation and ICC indexes required for significance.

The findings about the roughly symmetrical distribution of partial correlation indexes in each analysis imply that only one factor, represented by the total score, accounts for most of the variance common to the items and subgroup standing. This outcome does not bear on the issue of whether other common factors exist that only run through the items themselves and not through the subgroup variable too. Such a pos-

Table 2

Frequency Distributions of Partial Correlation Index in Main Analyses of Differential Functioning $(n=80)$

\begin{tabular}{|c|c|c|c|c|}
\hline \multirow[b]{2}{*}{ Value } & \multicolumn{2}{|c|}{ Race Analyses } & \multicolumn{2}{|c|}{ Sex Analyses } \\
\hline & Males & Females & wites & Blacks \\
\hline .20 to .29 & 0 & 0 & 0 & \\
\hline .10 to & 3 & 7 & 6 & 5 \\
\hline .00 to .09 & 42 & 37 & 38 & 35 \\
\hline .00 to -.09 & 30 & 32 & 29 & 36 \\
\hline-.10 to.- .19 & 5 & 3 & 6 & 3 \\
\hline-.20 to -.29 & 0 & 1 & 1 & 1 \\
\hline \multicolumn{5}{|c|}{$\begin{array}{l}\text { Note. In the race analyses, positive indexes signify items favor } \\
\text { ing blacks and negative indexes items favoring whites; in the sex } \\
\text { analyses, positive indexes signify items favoring females and negam } \\
\text { hive indexes items favoring males. }\end{array}$} \\
\hline
\end{tabular}

Downloaded from the Digital Conservancy at the University of Minnesota, http://purl.umn.edu/93227. May be reproduced with no cost by students and faculty for academic use. Non-academic reproduction requires payment of royalties through the Copyright Clearance Center, http://www.copyright.com/ 
sibility is consistent with other research that points to the factorial complexity of the items (Powers \& Swinton, 1981).

\section{Pserdosamunte Amalyses}

The number of items identified as significant or nonsignificant by the indexes in the supplementary analyses of differential functioning involving the pseudosamples appear in Table 1.

In the analyses of race differences in performance for the basic sample of white males and the pseudosample of black males, the partial correlation indexes identified as significant fewer than one-tenth of the items. This proportion was substantially lower than the corresponding proportion of significant items identified by this index in the main analyses. The ICC and item difficulty indexes also identified less than one-tenth of the items, appreciably lower than the proportion for the ICC index in the main analyses but the same as the corresponding proportion for the item difficulty in. dex.

Similarly, in the analyses of sex differences in functioning for the pseudosample of black males and the pseudosample of black females, the partial correlation index identified as significant fewer than one-tenth of the items. This proportion was appreciably lower than the corresponding one for the items in the main analyses. The ICC and item difficulty indexes also identified less than one-tenth of the items, far below the corresponding proportion for the ICC index in the main analyses but identical to the proportion for the item difficulty index.

These results indicate that the significance tests for the partial correlation and ICC indexes were reasonably accurate, for the proportions of significant indexes in these analyses for pairs of samples of the same race and sex (white males) were close to the proportion expected on the basis of the significance level employed (.05). These findings about the actual proportions of significant indexes obtained by chance under- score the point that the substantial proportions of items identified by the partial correlation and ICC indexes in the main analyses were well in excess of chance and the low proportions identified by the item difficulty index in the main analyses were at the chance level.

\section{Correspondence between}

The number of items consistently identified as significant and nonsignificant by the different indexes together with the corresponding Kappa coefficients appear in Table 3 for the main analyses of differential functioning.

In the analyses of race differences in performance, none of the indexes significantly $(p>.05)$ agreed with each other in the items that they identified as significant. In the analy. ses of sex differences in functioning, in contrast, the partial correlation and ICC indexes closely agreed, for both races, in the items identified $(\mathrm{Kappa}=.62$ to $.79, p<.01)$, though the item difficulty index did not significantly $(p>.05)$ agree with the two others.

The striking divergence in agreement between the partial correlation and ICC indexes in the analyses of race and sex differences is noteworthy. It is puzzling that the two indexes largely measured the same thing in one kind of analysis but not in another. One speculation is that the ICC index identified in the analyses of race differences, but not in the analyses of sex differences, items that favored a subgroup at one ability level but favored a second subgroup at another ability level. Hence, the partial correlation index, not being sensitive to such effects, identified different items than the $\mathbb{I C C}$ index in the analyses of race differences, but both indexes identified many of the same items in the analyses of sex differences where these effects were absent. The complete lack of agreement between the items identified by the item difficulty index and by the others primarily reflects the disparities in the proportions of items identified as significant by these indexes. 
Table 3

Agreament in Identification of Items by Different Indexes in Main Analyses of Differential Functioning $(n=80)$

\begin{tabular}{|c|c|c|c|c|c|c|}
\hline \multirow[b]{3}{*}{ Indexes } & \multicolumn{3}{|c|}{ Race Analyses } & \multicolumn{3}{|c|}{ Sex Analyses } \\
\hline & \multicolumn{2}{|c|}{ Identification } & \multirow[b]{2}{*}{ Kappa } & \multicolumn{2}{|c|}{ Identification } & \multirow[b]{2}{*}{ Kappa } \\
\hline & Same & Different & & Same & Different & \\
\hline & \multicolumn{3}{|c|}{ Males } & \multicolumn{3}{|c|}{ Whites } \\
\hline \multicolumn{7}{|l|}{ Partial Correlation } \\
\hline ys. Item Difficulty & 44 & 36 & $\infty .02$ & 50 & 30 & .18 \\
\hline \multicolumn{7}{|l|}{ Partial Correlation } \\
\hline ys. ICC & 44 & 36 & .00 & 72 & 8 & $.79^{*}$ \\
\hline \multicolumn{7}{|l|}{ Item Difficulty } \\
\hline \multirow[t]{2}{*}{ vs. ICC } & 68 & 12 & .28 & 54 & 26 & 19 \\
\hline & \multicolumn{3}{|c|}{ Females } & \multicolumn{3}{|c|}{ Blacks } \\
\hline \multicolumn{7}{|l|}{ Partial Corcelation } \\
\hline vs. Item Difficulty & 40 & 40 & .00 & 62 & 18 & .26 \\
\hline \multicolumn{7}{|l|}{ Partial Correlation } \\
\hline vs. ICC & 44 & 36 & .09 & 69 & 11 & $.62^{3 \pi}$ \\
\hline \multicolumn{7}{|l|}{ Item Difficulty } \\
\hline vs. ICC & 57 & 23 & .00 & 65 & 15 & .14 \\
\hline
\end{tabular}

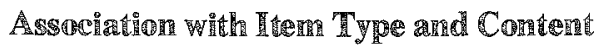

The number of items identified as significant and nonsignificant by the indexes in the main analyses of differential functioning for whites and blacks are classified by item type and item content in Table 4 . The $\chi^{2}$ s also appear in this table. $\left(\chi^{2}\right.$ s were not computed for the item difficulty index because of the small number of items identified as significant.) The corresponding stafistics for the analyses of differential performance for males and females appear in Table 5.

In the analyses of race differences in performance, the items identified by the partial correlation index were significantly $(p<.05)$ associated, for males only, with item type and content. The relationship with item type was mainly due to large numbers of Antonyms and small numbers of Reading Comprehension items with positive indexes, which represent functioning that favors blacks. The link with item content was primarily due to large numbers of Aesthetic-
Philosophical and Human Relationships items as well as small numbers of World of Practical Affairs items with positive indexes. None of the associations for items identified by the $\mathbb{I C C}$ index were significant $(p>.05)$.

In the analyses of sex differences in functioning, the items identified by the partial correlation index were significantly $(p<.05)$ associated, for blacks only, with item type and, for whites as well as blacks, with content. The connection with item type was mainly due to large numbers of Reading Comprehension items with positive indexes, which reflect performance that favors females; and small numbers of Reading Comprehension items and large numbers of Analogies with negative indexes, which represent functioning that favors males. The relationship with item content, for whites, was mainly due to large numbers of Female items with positive indexes. For blacks, the link with item content was also primarily due to large numbers of Female items with positive indexes as well as to 


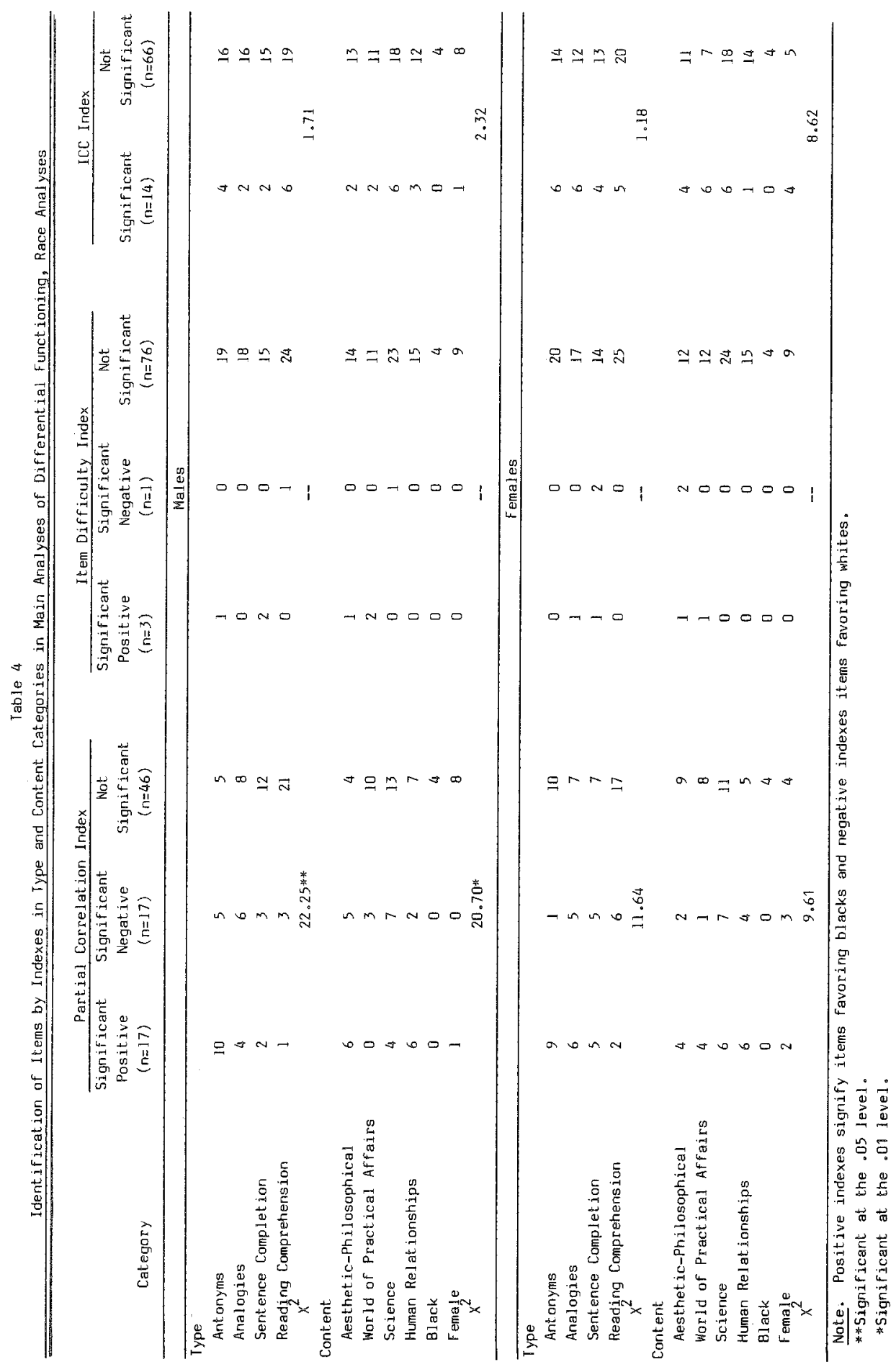

Downloaded from the Digital Conservancy at the University of Minnesota, http://purl.umn.edu/93227. May be reproduced with no cost by students and faculty for academic use. Non-academic reproduction requires payment of royalties through the Copyright Clearance Center, http://www.copyright.com/ 


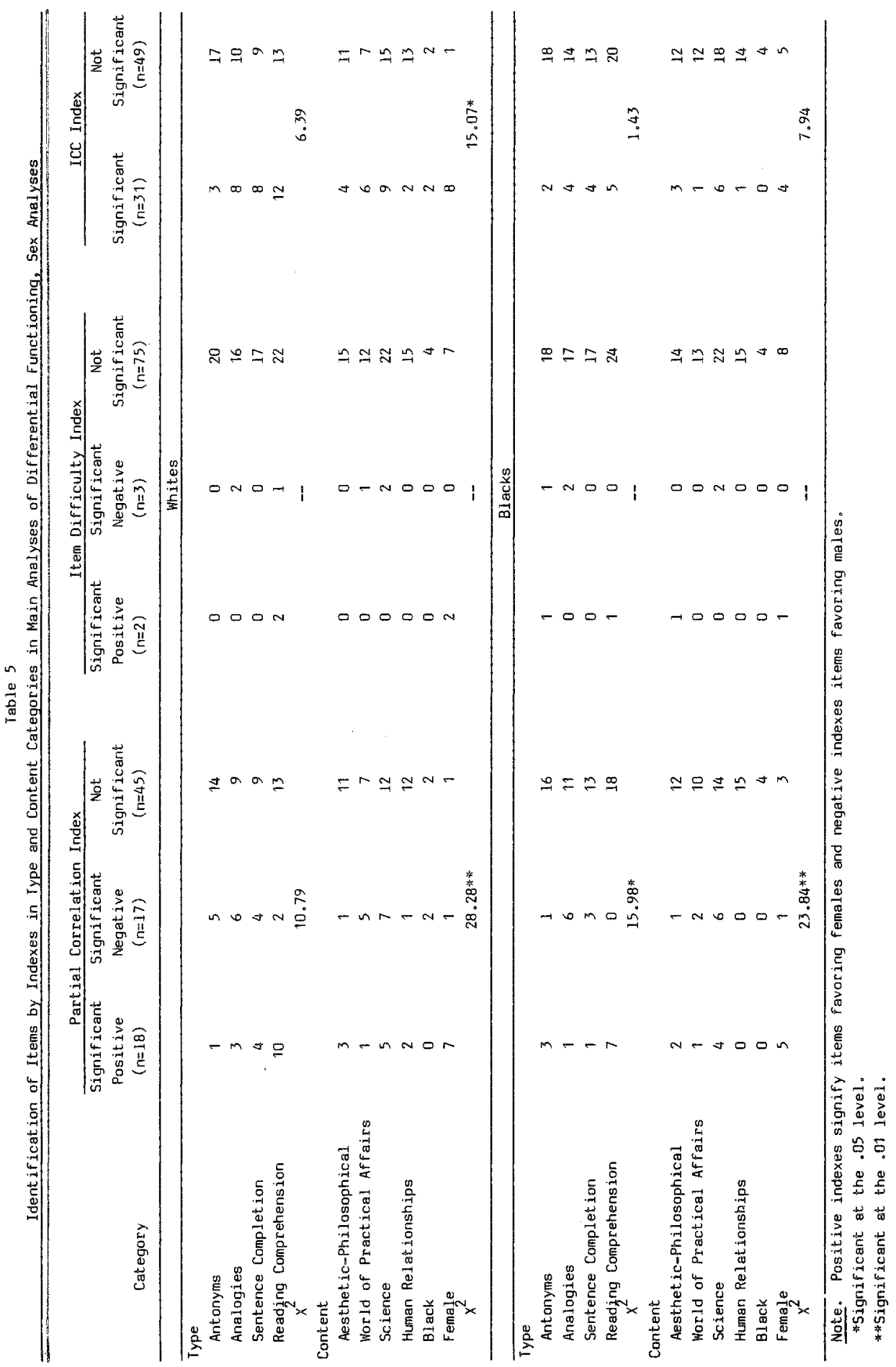

Downloaded from the Digital Conservancy at the University of Minnesota, http://purl.umn.edu/93227. May be reproduced with no cost by students and faculty for academic use. Non-academic reproduction requires payment of royalties through the Copyright Clearance Center, http://www.copyright.com/ 
large numbers of Science items with negative indexes. The items identified by the ICC index were significantly $(p<.05)$ associated, for whites only, with item content. This link was mainly due to the identification of large numbers of $\mathrm{Fe}$ male items and small numbers of Human Relationships items. None of the associations with item type for items identified by this index were significant $(p>.05)$ 。

The overall lack of association between the items identified by the indexes in the analyses of race and sex differences and the items' type and content is congruent with the sparse findings in previous work discussed earlier. A major exception is that the partial correlation index consistently identified, for both races, items with female content which favored females. However, apart from the finding, obtained for blacks only, that this same index identified items with science content which favored males, the results did not confirm other relationships suggested by earlier research: items with practical content favoring males and items with human relationships content favoring females.

The tendency for the limited associations observed between the items that were identified and their type and content to center around the partial correlation index and content deserves attention. The involvement of the partial correlation index provides some support for the validity of this procedure. In addition, the link with content indicates that this classification scheme is not only meaningful but also has some value in identifying differentially performing items; the contrasting lack of association with item type implies either that this means of categorizing items is not sensible or that this characteristic is unrelated to differential functioning in these items.

\section{Conclusion}

The present evidence concerning the partial correlation index's agreement with the ICC index and associations with item content, along with its inherent ease of calculation, applicabil- ity to small samples, flexibility in accommodato ing different kinds of subgroups, and provision for an appraisal of statistical significance and magnitude of effect, suggest that this measure may be useful in future work in this area.

\section{Ieterences}

Angoff, W. H., \& Ford, S. F. Item-race interaction on a test of scholastic aptitude. Journal of Educational Measurement, 1973, 10, 95-106.

Cleary, T. A., \& Hilton, T. L. An investigation of item bias. Educational and Psychological Measurement, 1968, 28, 61-75.

Coffman, W. E. Sex differences in responses to items in an aptitude test. In E. M. Huddleston (Ed.), Eighteenth Yearbook, National Council on Measurement in Education. Ames IA: National Council on Measurement in Education, 1961.

Cohen, J. A. A coefficient of agreement for nominal scales. Educational and Psychological Measurement, 1960, 20, 37-46.

Conrad, L., Trismen, D., \& Miller, R. (Eds.). Graduate Record Examinations technical manual. Princeton NJ: Educational Testing Service, 1977.

Darlington, R. B. Another look at "cultural fairness." Journal of Educational Measurement, $1971,8,71-82$.

Donlon, T. F., \& Angoff, W. H. The Scholastic Aptitude Test. In W. H. Angoff (Ed.), The College Board admissions testing program: A technical report on research and development activities relat. ing to the Scholastic Aptitude Test and achievement tests. New York: College Entrance Examina. tion Board, 1971.

Donlon, T. F., Hicks, M. M., \& Wallmark, M. M. Sex differences in item responses on the Graduate Record Examination. Applied Psychological Measurement, 1980, 4, 9-20.

Flaugher, R. L. The many definitions of test bias. American Psychologist, 1978, 33, 671-679.

Fleiss, J. L., Cohen, J., \& Everitt, B. S. Large sample standard errors of Kappa and weighted Kappa. Psychological Bulletin, 1969, 72, 323-327.

Guilford, J. P. Fundamental statistics in psychology and education (4th ed.). New York: McGraw-Hill, 1965.

Harman, H. H. Modern factor analysis (3rd ed.). Chicago: University of Chicago Press, 1976.

Jensen, A. R. Bias in mental testing. New York: Free Press, 1980.

Lord, F. M. A study of item bias, using item characteristic curve theory. In Y. H. Poortinga (Ed.), 
Basic problems in cross-cultural research. Amsterdam: Swets \& Zeitlinger, 1977. (a)

Lord, F. M. Personal communication, November 29 , 1977. (b)

Lord, F. M. Personal communication, July 13, 1979.

Lord, F. M. Applications of item response theory to practical testing problems. Hillsdale NJ: Erlbaum, 1980.

Lord, F. M., \& Novick, M. R. Statistical theories of mental test scores. Reading MA: Addison-Wesley, 1968.

Magnusson, D. Test theory. Reading MA: AddisonWesley, 1967.

Merz, W. R. Test fairness and test bias: A review of procedures. In M. J. Wargo \& D. R. Green (Eds.), Achievement testing of disadvantaged and minority students for educational program evaluation. Monterey CA: CTB/McGraw-Hill, 1978.

Petersen, N. S. Bias in the selection rule-Bias in the test. In L. J. T. van der Kamp, W. F. Langerak, \& D. N. M. de Gruijter (Eds.), Psychometrics for educational debates. New York: Wiley, 1980.

Powers, D. E., \& Swinton, S. S. Extending the measurement of graduate admission abilities beyond the verbal and quantitative domains. Applied Psy chological Measurement, 1981, 5, 141-158.

Rudner, L. M., Getson, P. R., \& Knight, D. L. Biased item detection techniques. Journal of Educational Statistics, 1980, 5, 213-233.

Shepard, L. A. Identifying bias in test items. In B. F. Green (Ed.), Issues in testing: Coaching, disclosure, and ethnic bias. San Francisco: Jossey-Bass, 1981.

Wood, R. L., Wingersky, M. S., \& Lord, F. M. LOGIST - A computer program for estimating examinee ability and item characteristic curve parameters (ETS RM 76-6). Princeton NJ: Educational Testing Service, 1976.

\section{Ackiow ledgments}

This study was supported in part by the Graduate Record Examinations Board. Portions of this article were presented at the meeting of the Society of Multivariate Experimental Psychology, Los Angeles, November 1979. Thanks are due Douglas N. Jackson, Frederic M. Lord, Ledyard R. Tucker, and Madeline $M$. Wallmark for advising about the research design and statistical analysis; Frans C. van der Lee for arranging the retrieval of the data; Henrietta Gallagher for statistical calculating: Satya P. Agarwal, Katherine Kornhauser, Barbara J. Phillips, Alfred Rogers, Caroline Roth, and Marilyn S. Wingersky for computer programming; and Donald L. Alderman, Robert A. Altman, William H. Angoff, Mary Jo Clark, Nancy S. Cole, Thomas F. Donlon, Bert $F$. Green, Neal M. Kingston, Frederic M. Lord, and Cheryl $L$. Wild for critically reviewing drafts of this article.

\section{Author's Address}

Send requests for reprints or further information to Lawrence J. Stricker, Educational Testing Service, Princeton NJ 08541.

\section{Appendix}

The following proof that the partial correlation $\varrho_{i s \cdot \tau}$ is zero when an item is unbiased was derived by Lord (1979):

If an item $(i)$ is unbiased, then for any fixed level of ability $\tau, \varrho_{i s \mid \tau}=0$, where $\varrho_{i s \mid \tau}$ is the prod. uct-moment correlation between the item and subgroup standing for examinees at ability level $\tau$. Thus, $o_{i s \mid \tau}=0$, where $o_{i s \mid \tau}$ is the covariance between the item and subgroup standing at a particular level. Because subtracting a constant from a variable does not change its covariance,

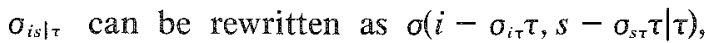
where $\sigma_{i \tau}$ is the total-group covariance between the item and ability and $o_{s \tau}$ is the total-group co. variance between subgroup standing and ability. Therefore, $\sigma\left(i-\sigma_{i \tau} \tau, s-\sigma_{s \tau} \mid \tau\right)=0$. Averaging across ability levels, the total-group covariance $\sigma\left(i-\sigma_{i \tau} \tau, s-\sigma_{s \tau} \tau\right)=0$. If the scale for $\tau$ is chosen so that $\tau$ has zero mean and unit variance, then $\hat{i}=\bar{i}+\sigma_{i \tau} \tau$ and $\hat{s}=\bar{s}+\sigma_{s \tau} \tau$, where $\hat{i}$ and $\hat{s}$ are the item and subgroup standing scores, respectively, predicted from ability. Thus, of $\left.i-\sigma_{i \tau} \tau, s-o_{s t} \tau\right)=o(i-\hat{i}, s-\hat{s})$. It is well known that $\mathrm{Q}(i-\hat{i}, s-\hat{s})$ is $\varrho_{i s \cdot}$. Therefore, $\varrho_{i s \cdot \tau}=0$. 